\title{
Universal Superspace Unitary Operator for Some Interesting Abelian Models: Superfield Approach
}

\author{
T. Bhanja, ${ }^{1}$ N. Srinivas, ${ }^{1}$ and R. P. Malik ${ }^{1,2}$ \\ ${ }^{1}$ Physics Department, Centre of Advanced Studies, Banaras Hindu University, Varanasi, Uttar Pradesh 221005 , India \\ ${ }^{2}$ DST Centre for Interdisciplinary Mathematical Sciences, Faculty of Science, Banaras Hindu University, Varanasi 221 005, India \\ Correspondence should be addressed to T. Bhanja; tapobroto.bhanja@gmail.com
}

Received 20 November 2015; Accepted 31 December 2015

Academic Editor: Sally Seidel

Copyright (c) 2016 T. Bhanja et al. This is an open access article distributed under the Creative Commons Attribution License, which permits unrestricted use, distribution, and reproduction in any medium, provided the original work is properly cited. The publication of this article was funded by $\mathrm{SCOAP}^{3}$.

\begin{abstract}
Within the framework of augmented version of superfield formalism, we derive the superspace unitary operator and show its usefulness in the derivation of Becchi-Rouet-Stora-Tyutin (BRST) and anti-BRST symmetry transformations for a set of interesting models for the Abelian 1-form gauge theories. These models are (i) a one (0+1)-dimensional (1D) toy model of a rigid rotor, (ii) the two (1+1)-dimensional (2D) modified versions of the Proca and anomalous Abelian 1-form gauge theories, and (iii) the 2D self-dual bosonic gauge field theory. We provide, in some sense, the alternatives to the horizontality condition (HC) and the gauge invariant restrictions (GIRs) in the language of the above superspace (SUSP) unitary operator. One of the key observations of our present endeavor is the result that the SUSP unitary operator and its Hermitian conjugate are found to be the same for all the Abelian models under consideration (including the 4D interacting Abelian 1-form gauge theories with Dirac and complex scalar fields which have been discussed earlier). Thus, we establish the universality of the SUSP operator for the above Abelian theories.
\end{abstract}

\section{Introduction}

The usual superfield approach [1-8] to Becchi-Rouet-StoraTyutin (BRST) formalism is a geometrically rich and physically very intuitive method which sheds light on the physical interpretation of the nilpotency and absolute anticommutativity property of the proper (anti-)BRST symmetries. These properties are sacrosanct because the nilpotency property encodes the fermionic nature of the (anti-)BRST symmetries and the absolute anticommutativity property represents the linear independence of the BRST and anti-BRST symmetries. In addition, the above superfield approach also leads to the derivation of the precise form of the (anti-)BRST symmetries and associated Curci-Ferrari (CF) condition in the description of the $p$-form $(p=1,2,3, \ldots)$ (non-)Abelian gauge theories (see, e.g., $[4-6,9,10]$, for details). However, this approach is useful only in the derivation of the (anti-)BRST symmetries for the $p$-form gauge and associated (anti-)ghost fields of a given $p$-form gauge theory. It does not shed any light on the derivation of the (anti-)BRST symmetries that are associated with the matter fields in a given interacting $p$-form gauge theory.

The above superfield approach has been consistently generalized so as to derive the (anti-)BRST symmetries for the gauge, associated (anti-)ghost and matter fields of a given interacting (non-)Abelian 1-form gauge theory by invoking the horizontality condition (HC) and the gauge (i.e., (anti-)BRST) invariant restrictions (GIRs) on the superfields defined on the $(\mathrm{D}, 2)$-dimensional supermanifold corresponding to a given $\mathrm{D}$-dimensional interacting (non-)Abelian 1 -form gauge theory defined on the $\mathrm{D}$-dimensional flat Minkowski spacetime ordinary manifold (see, e.g., [1114]). The consistently generalized version of the superfield approach to BRST formalism [4-6] has been christened as the augmented version of superfield approach to BRST formalism in our earlier works [11-14].

In the seminal works [4-6], a superspace (SUSP) unitary operator has been introduced to derive the (anti-)BRST symmetries associated with the gauge field, (anti-)ghost fields, and a generic matter field in the context of the superfield 
approach to BRST formalism. This SUSP unitary operator has been introduced so as to maintain the explicit group structure in the SUSP transformation space of the fields of a given $4 \mathrm{D}$ non-Abelian 1-form gauge theory. However, the precise expressions for this SUSP unitary operator and its Hermitian conjugate have not been explicitly derived mathematically. Rather, these expressions have been chosen intelligently. The Hermitian conjugate operator (corresponding to a SUSP unitary operator) has been derived after imposing some Hermitian conjugation conditions from outside on the fields as well as the Grassmannian parameters of the superfield version of the theory. The above SUSP operators, besides maintaining the group structure, provide the alternatives to the $\mathrm{HC}$ and GIRs as we will see in our further discussions.

In a very recent couple of papers $[15,16]$, we have been able to derive explicitly the expressions for the SUSP unitary operator and its Hermitian conjugate without imposing any outside Hermitian conjugation conditions on the fields and/or the Grassmannian variables of the supersymmetrized version of the interacting 4D Abelian and (non-)Abelian 1form gauge theories. In the former case, we have discussed the QED with Dirac and complex scalar fields coupled with the Abelian 1-form gauge field [15] and, in the latter case, we have considered the $4 \mathrm{D}$ non-Abelian interacting theory with Dirac fields. The purpose of our present endeavor is to show the universality of the above SUSP unitary operator and its Hermitian conjugate in the description of the 1D and 2D Abelian 1-form gauge theories of different kinds where the covariant derivatives are not explicitly defined. For this purpose, we take into consideration the $1 \mathrm{D}$ toy model of a rigid rotor and the 2D modified versions of the Proca as well as the anomalous gauge theories and the $2 \mathrm{D}$ self-dual bosonic theory. These models are very interesting Abelian 1-form theories in one and two dimensions of spacetime because these have been proven to provide the physical examples of Hodge theory (see, e.g., [17-23]) where there exist many interesting internal symmetries (and corresponding conserved charges). To be precise, the models under considerations respect the proper (anti-)BRST and (anti-)co-BRST symmetry transformations, a unique bosonic symmetry transformation, and the ghost-scale symmetry transformations which together provide the physical realizations of the cohomological operators of differential geometry.

In our present investigation, we have applied the augmented version of superfield/supervariable formalism to derive the (anti-)BRST symmetries for the 2D and 1D Abelian 1-form gauge theories and expressed these results in terms of the SUSP unitary operators. We have shown that the mathematical form of the SUSP unitary operator and its Hermitian conjugate is universal for the $1 \mathrm{D}, 2 \mathrm{D}$, and $4 \mathrm{D} 1-$ form gauge theories. In fact, we have obtained the universal form of the above operators which maintain the $U(1)$ group structure in the SUSP transformation space of the gauge variables/fields and other variables/fields of these theories. Furthermore, the SUSP unitary operators have also been shown to provide the alternatives to the $\mathrm{HC}$ and GIRs that enable us to derive the full set of proper (anti-)BRST symmetry transformations for all the variables/fields of a given theory. The universality of the mathematical form of the
SUSP unitary operator (and its Hermitian conjugate) is one of the highlights of our present investigation where we have considered different kinds of Abelian 1-form gauge theories.

Our present investigation is essential because it is motivated by the following key factors. First and foremost, we have demonstrated the universality of the SUSP unitarity operator (and its Hermitian conjugate) for all the Abelian 1form gauge theories defined on the $1 \mathrm{D}, 2 \mathrm{D}$, and $4 \mathrm{D}$ flat background Minkowski spacetime manifolds. This observation is one of the decisive features of our present investigation. Second, the existence of the SUSP unitarity operator (and its Hermitian conjugate) provides the alternatives to the $\mathrm{HC}$ and the GIRs where the group structure is very explicitly and elegantly maintained. This is a very nice feature of the SUSP unitary operator and its Hermitian conjugate. This group structure is somewhat hidden when we exploit the potential and power of the HC and the GIRs. Third, the Abelian models under considerations are interesting because they provide a set of tractable examples of the Hodge theory within the framework of BRST formalism [17-23]. Finally, our present work is our modest first step towards our central goal of providing a theoretical generality for the existence of the SUSP unitarity operator and its Hermitian conjugate for the interacting non-Abelian gauge theories as well.

The material of our present investigation is organized as follows. In Section 2, to set up the convention and notations, we discuss briefly the (anti-)BRST symmetries in the Lagrangian formulation for the one $(0+1)$-dimensional (1D) toy model of a rigid rotor and $2 \mathrm{D}$ modified versions of the Proca theory as well as the anomalous gauge theory and 2D self-dual bosonic field theory; Section 3 is devoted to a concise description of the HC and the GIRs which enable us to derive the above (anti-)BRST symmetry transformations within the framework of augmented superfield formalism. Section 4 deals with the derivation of the results, obtained in Section 3, in terms of the superspace unitarity operator and its Hermitian conjugate. Finally, we summarize our key results, make some concluding remarks, and point out a few future directions for further investigations in Section 5.

General Notations and Convention. We denote the (anti-)BRST symmetries for all the Abelian models by the symbols $s_{(a) b}$ in our present endeavor. For the 2D theories, we adopt the convention such that the 2D background Minkowski spacetime manifold is endowed with the metric $\eta_{\mu \nu}$ with the signatures $(+1,-1)$ so that the dot product between two nonnull vectors $A_{\mu}$ and $B_{\mu}$ is denoted by $A \cdot B=A_{\mu} B^{\mu}=\eta_{\mu \nu} A^{\mu} B^{\nu}=A_{0} B_{0}-A_{i} B_{i}$, where the Greek indices $\mu, \nu, \lambda, \ldots=0,1$ stand for the spacetime directions and the Latin indices $i, j, k, \ldots=1$ correspond to the space direction only. We choose the 2D Levi-Civita tensor $\varepsilon_{\mu \nu}$ (with $\varepsilon_{01}=+1=-\varepsilon^{01}$ ) which satisfy $\varepsilon_{\mu \nu} \varepsilon^{\mu \nu}=2$ !, $\varepsilon_{\mu \nu} \varepsilon^{\nu \lambda}=\delta_{\mu}^{\lambda}$, and $\varepsilon_{\mu \nu} \varepsilon^{\mu \lambda}=-\delta_{\nu}^{\lambda}$. We denote the scalar field by $\phi(x, t)$ in the cases of the 2D modified versions of Proca theory, anomalous gauge theory, and the 2D self-dual chiral bosonic theory. The corresponding superfield has been denoted by $\Phi(x, \theta, \bar{\theta})$ within the framework of superfield formalism. 


\section{Preliminaries: (Anti-)BRST Symmetries}

We discuss here, first of all, the (anti-)BRST symmetries $s_{(a) b}$ in the context of a 1D toy model of a rigid rotor (with mass $m=1$ ) which is described by the following first-order (anti-)BRST invariant Lagrangian (see, e.g., [17-19, 24], for details):

$$
\begin{aligned}
L_{b}= & \dot{r} p_{r}+\dot{\vartheta} p_{\vartheta}-\frac{p_{\vartheta}^{2}}{2 r^{2}}-\lambda(r-a)+B\left(\dot{\lambda}-p_{r}\right)+\frac{B^{2}}{2} \\
& -i \dot{\bar{C}} \dot{C}+i \bar{C} C
\end{aligned}
$$

where $(r, \vartheta)$ is a pair of polar coordinates, the pair $\left(p_{r}, p_{\vartheta}\right)$ corresponds to the conjugate momenta with respect to $(r, \vartheta)$, $\lambda(t)$ is the Lagrange multiplier that turns out to be the analogue of the "gauge" variable, $B(t)$ is the NakanishiLautrup type auxiliary variable, and the fermionic $\left(C^{2}=\right.$ $\bar{C}^{2}=0, C \bar{C}+\bar{C} C=0$ ) (anti-)ghost variables $(\bar{C}) C$ are needed for the sake of unitarity in our theory. The above Lagrangian (1) respects the following supersymmetric type off-shell nilpotent $\left(s_{(a) b}^{2}=0\right)$ and absolutely anticommuting $\left(s_{b} s_{a b}+s_{a b} s_{b}=0\right)\left(\right.$ anti-)BRST symmetry transformation $s_{(a) b}$ :

$$
\begin{aligned}
s_{b}\left(r, \vartheta, p_{\vartheta}, B, C\right) & =0, \\
s_{b} \lambda & =\dot{C}, \\
s_{b} p_{r} & =-C, \\
s_{b} \bar{C} & =i B, \\
s_{a b}\left(r, \vartheta, p_{\vartheta}, B, \bar{C}\right) & =0, \\
s_{a b} \lambda & =\dot{\bar{C}}, \\
s_{a b} p_{r} & =-\bar{C}, \\
s_{a b} C & =-i B .
\end{aligned}
$$

As a consequence, the action integral $S=\int d t L_{b}$ remains invariant under $s_{(a) b}$ (because $s_{b} L_{b}=(\partial / \partial t)[B \dot{C}-(r-$ a)C], $\left.s_{a b} L_{b}=(\partial / \partial t)[B \dot{\bar{C}}-(r-a) \bar{C}]\right)$. In our discussion, all the variables are function of the evolution parameter $t$ and the pair $(\dot{r}=d r / d t, \dot{\vartheta}=d \vartheta / d t)$ stands for the generalized radial and angular velocities.

We now focus on the Stuckelberg modified version of the 2D Proca theory whose proper (anti-)BRST invariant Lagrangian density is (see, e.g., [20, 21], for details)

$$
\begin{aligned}
\mathscr{L}_{B}= & -\frac{1}{4} F_{\mu \nu} F^{\mu \nu}+\frac{m^{2}}{2} A_{\mu} A^{\mu}+\frac{1}{2} \partial_{\mu} \phi \partial^{\mu} \phi-m A_{\mu} \partial^{\mu} \phi \\
& +B(\partial \cdot A+m \phi)+\frac{B^{2}}{2}-i \partial_{\mu} \bar{C} \partial^{\mu} C+i m^{2} \bar{C} C,
\end{aligned}
$$

where $F_{\mu \nu}=\partial_{\mu} A_{\nu}-\partial_{\nu} A_{\mu}$ is the curvature tensor that is derived from the curvature 2-form $F^{(2)}=d A^{(1)} \equiv\left[\left(d x^{\mu} \wedge\right.\right.$ $\left.\left.d x^{\nu}\right) / 2 !\right] F_{\mu \nu}$. Here, $d=d x^{\mu} \partial_{\mu}$ is the exterior derivative (with $\left.d^{2}=0\right)$ and the 1 -form $A^{(1)}=d x^{\mu} A_{\mu}$ defines the $U(1)$ vector potential $A_{\mu}$. The Stueckelberg field $\phi$ (with mass $m$ ) has been invoked to convert the second-class constraints of the $2 \mathrm{D}$ Proca theory (with mass $m$ ) into the first-class constraints. We have the (anti-)ghost fields $(\bar{C}) C$, too, in our BRST invariant theory for the proof of unitarity. It is elementary to check that the following off-shell nilpotent $\left(s_{(a) b}^{2}=0\right)$ and absolutely anticommuting $\left(s_{b} s_{a b}+s_{a b} s_{b}=0\right)$ (anti-)BRST symmetry transformations $s_{(a) b}$

$$
\begin{aligned}
s_{b} A_{\mu} & =\partial_{\mu} C, \\
s_{b} \phi & =m C, \\
s_{b} \bar{C} & =i B, \\
s_{b}\left(B, F_{\mu \nu}, C\right) & =0, \\
s_{a b} A_{\mu} & =\partial_{\mu} \bar{C}, \\
s_{a b} \phi & =m \bar{C}, \\
s_{a b} C & =-i B, \\
s_{a b}\left(B, F_{\mu \nu}, \bar{C}\right) & =0,
\end{aligned}
$$

leave the action integral $S=\int d^{2} x \mathscr{L}_{B}$ invariant for the physically well-defined fields which vanish off at $x= \pm \infty$ because $s_{b} \mathscr{L}_{B}=\partial_{\mu}\left(B \partial^{\mu} C\right)$ and $s_{a b} \mathscr{L}_{B}=\partial_{\mu}\left(B \partial^{\mu} \bar{C}\right)$.

The next Abelian model of interest is the 2D self-dual bosonic field theory whose precise (anti-)BRST invariant Lagrangian density is (see, e.g., $[22,23]$ )

$$
\begin{aligned}
\mathscr{L}_{B}^{(s)}= & \frac{1}{2} \dot{\phi}^{2}-\frac{1}{2} \dot{v}^{2}+\dot{v}\left(v^{\prime}-\phi^{\prime}\right)+\lambda\left[\dot{\phi}-\dot{v}+v^{\prime}-\phi^{\prime}\right] \\
& -\frac{1}{2}\left(\phi^{\prime}-v^{\prime}\right)^{2}+B(\dot{\lambda}-v-\phi)+\frac{B^{2}}{2}-\dot{\bar{C}} \dot{C} \\
& +2 i \bar{C} C,
\end{aligned}
$$

where $\dot{\phi}=\partial \phi / \partial t, \dot{v}=\partial v / \partial t, \dot{\lambda}=\partial \lambda / \partial t$, and so forth are the generalized "velocities" with respect to the evolution parameter $t$ of our present theory. We also follow the notation: $\phi^{\prime}=\partial \phi / \partial x, v^{\prime}=\partial v / \partial x$ which is nothing but the single space derivative on the $2 \mathrm{D}$ self-dual field $\phi(x, t)$ and Wess-Zumino field $v(x, t)$. As explained earlier, $B(x, t)$ is the Nakanishi-Lautrup type auxiliary field and $(\bar{C}) C$ are the (anti-)ghost fields. It is straightforward to check that, under the following off-shell nilpotent and absolutely anticommuting (anti-)BRST symmetry transformations (see, e.g., [22, 23], for details)

$$
\begin{aligned}
s_{a b} \lambda & =\dot{\bar{C}}, \\
s_{a b} \phi & =-\bar{C}, \\
s_{a b} v & =-\bar{C}, \\
s_{a b} C & =-i B, \\
s_{a b}(B, \bar{C}) & =0,
\end{aligned}
$$




$$
\begin{aligned}
s_{b} \lambda & =\dot{C}, \\
s_{b} \phi & =-C, \\
s_{b} v & =-C, \\
s_{b} \bar{C} & =i B, \\
s_{b}(B, C) & =0,
\end{aligned}
$$

the Lagrangian density $\mathscr{L}_{B}^{(s)}$ transforms as follows: $s_{b} \mathscr{L}_{B}^{(s)}=$ $(\partial / \partial t)[B \dot{C}], s_{a b} \mathscr{L}_{B}^{(s)}=(\partial / \partial t)[B \dot{\bar{C}}]$, thereby leaving the action integral $S=\int d^{2} x \mathscr{L}_{B}^{(s)}$ invariant for the physically welldefined fields which vanish off at $t= \pm \infty$.

Finally, we concentrate on the modified version of a $2 \mathrm{D}$ anomalous Abelian 1-form gauge theory (see, e.g., [25]) in its bosonized version. In this context, the (anti-)BRST invariant Lagrangian density for this Abelian system (with electric charge $e=1$ ) is [25]

$$
\begin{aligned}
\mathscr{L}_{B}^{(a)}= & -\frac{1}{4} F^{\mu \nu} F_{\mu \nu}+\frac{1}{2} \partial_{\mu} \phi \partial^{\mu} \phi+\frac{a}{2} A_{\mu} A^{\mu} \\
& +\left(\eta^{\mu \nu}-\varepsilon^{\mu \nu}\right) \partial_{\mu} \phi A_{\nu} \\
& +\sigma\left[(a-1)(\partial \cdot A)+\varepsilon^{\mu \nu} \partial_{\mu} A_{\nu}\right] \\
& +\frac{(a-1)}{2} \partial_{\mu} \sigma \partial^{\mu} \sigma+B(\partial \cdot A)+\frac{B^{2}}{2}-i \partial_{\mu} \bar{C} \partial^{\mu} C
\end{aligned}
$$

where the Abelian 1-form $A^{(1)}=d x^{\mu} A_{\mu}$ defines the vector potential $A_{\mu}$ and the curvature 2-form $F^{(2)}=d A^{(1)}$ defines $F_{\mu \nu}=\partial_{\mu} A_{\nu}-\partial_{\nu} A_{\mu}$ (which has only electric field (i.e., $F_{01}=$ $\left.E \equiv-\varepsilon^{\mu \nu} \partial_{\mu} A_{\nu}\right)$ as its existing component in 2D). In the above, $a$ is the parameter of ambiguity in the regularization of the fermionic determinant when the $2 \mathrm{D}$ chiral Schwinger model (with electric charge $e=1$ ) is bosonized in terms of the scalar field $\phi$. A bosonic field $\sigma(x)$ has been introduced to convert the second-class constraints of the chiral Schwinger model into the first-class constraints. As discussed earlier, the proper (anti-)BRST invariant Lagrangian density (7) contains the fermionic (anti-)ghost fields $(\bar{C}) C$ and the NakanishiLautrup type auxiliary field $B(x)$. Under the following offshell nilpotent $\left(s_{(a) b}^{2}=0\right)$ and absolutely anticommuting $\left(s_{b} s_{a b}+s_{a b} s_{b}=0\right)$ (anti-)BRST symmetry transformations

$$
\begin{aligned}
s_{a b} A_{\mu} & =\partial_{\mu} \bar{C}, \\
s_{a b} C & =-i B, \\
s_{a b} \phi & =-\bar{C}, \\
s_{a b} \sigma & =\bar{C}, \\
s_{a b}\left[B, \bar{C}, F_{\mu \nu}\right] & =0, \\
s_{b} A_{\mu} & =\partial_{\mu} C, \\
s_{b} \bar{C} & =i B,
\end{aligned}
$$

$$
\begin{aligned}
s_{b} \phi & =-C, \\
s_{b} \sigma & =C, \\
s_{b}\left[B, C, F_{\mu \nu}\right] & =0,
\end{aligned}
$$

the Lagrangian density (7) transforms as follows:

$$
\begin{aligned}
& s_{a b} \mathscr{L}_{B}^{(a)}=\partial_{\mu}\left[B \partial^{\mu} \bar{C}+(a-1)\left(\sigma \partial^{\mu} \bar{C}+A^{\mu} \bar{C}\right)\right. \\
& \left.\quad-\varepsilon^{\mu \nu}\left(\phi \partial_{\nu} \bar{C}-A_{\nu} \bar{C}\right)\right] \\
& s_{b} \mathscr{L}_{B}^{(a)}=\partial_{\mu}\left[B \partial^{\mu} C+(a-1)\left(\sigma \partial^{\mu} C+A^{\mu} C\right)\right. \\
& \left.\quad-\varepsilon^{\mu \nu}\left(\phi \partial_{\nu} C-A_{\nu} C\right)\right] .
\end{aligned}
$$

As a consequence, the action integral $S=\int d^{2} x \mathscr{L}_{B}^{(a)}$ remains invariant under the nilpotent (anti-)BRST symmetry transformations (8) for the physically well-defined fields which vanish off at $x= \pm \infty$ when we apply the Gauss divergence theorem.

\section{3. (Anti-)BRST Symmetries: Superfield Approach}

In this section, we derive the proper (i.e., off-shell nilpotent and absolutely anticommuting) (anti-)BRST symmetry transformations (for all the Abelian models under consideration) by exploiting the potential and power of $\mathrm{HC}$ and GIRs. Towards this goal in mind, first of all, we generalize the basic variables $\lambda(t), C(t), \bar{C}(t)$ of the $1 \mathrm{D}$ rigid rotor onto a $(1,2)$ dimensional supermanifold, parametrized by the superspace coordinate $Z^{M}=(t, \theta, \bar{\theta})$, as follows (see, e.g., [17-19], for details):

$$
\begin{aligned}
\lambda(t) & \longrightarrow \\
\Lambda(t, \theta, \bar{\theta}) & =\lambda(t)+\theta \bar{R}(t)+\bar{\theta} R(t)+i \theta \bar{\theta} S(t), \\
C(t) & \longrightarrow \\
F(t, \theta, \bar{\theta}) & =C(t)+i \theta \bar{B}_{1}(t)+i \bar{\theta} B_{1}(t)+i \theta \bar{\theta} s(t), \\
\bar{C}(t) & \longrightarrow \\
\bar{F}(t, \theta, \bar{\theta}) & =\bar{C}(t)+i \theta \bar{B}_{2}(t)+i \bar{\theta} B_{2}(t)+i \theta \bar{\theta} \bar{s}(t),
\end{aligned}
$$

where the secondary variables $(R, \bar{R}, s, \bar{s})$ and $\left(B_{1}, \bar{B}_{1}, B_{2}\right.$, $\left.\bar{B}_{2}, S\right)$ are fermionic and bosonic in nature because of the fermionic $\left(\theta^{2}=\bar{\theta}^{2}=0, \theta \bar{\theta}+\bar{\theta} \theta=0\right)$ nature of the pair of Grassmannian variables $(\theta, \bar{\theta})$ of the superspace coordinate $Z^{M}=(t, \theta, \bar{\theta})$. To determine the exact form of the above secondary variables in terms of the basic and auxiliary variables of the Lagrangian (1) for the 1D rigid rotor, we have to exploit the potential and power of the HC defined on the $(1,2)$-dimensional supermanifold. 
We define an Abelian 1-form $\lambda^{(1)}=d t \lambda(t)$ so that a null 2 -form $d \lambda^{(1)}=0$ (where $d=d t \partial_{t}$ is the exterior derivative) could be constructed on a 1D ordinary manifold. This can be generalized onto an appropriate $(1,2)$-dimensional supermanifold as

$$
\begin{aligned}
d \lambda^{(1)} & \longrightarrow \\
\tilde{d} \tilde{\lambda}^{(1)} & \\
= & {\left[d t \partial_{t}+d \theta \partial_{\theta}+d \bar{\theta} \partial_{\bar{\theta}}\right] } \\
& \wedge[d t \Lambda(t, \theta, \bar{\theta})+d \theta \bar{F}(t, \theta, \bar{\theta})+d \bar{\theta} F(t, \theta, \bar{\theta})],
\end{aligned}
$$

where we have used the following generalizations

$$
\begin{aligned}
d & \longrightarrow \widetilde{d}=d t \partial_{t}+d \theta \partial_{\theta}+d \bar{\theta} \partial_{\bar{\theta}}, \\
\lambda^{(1)}(t) & \longrightarrow \\
\tilde{\lambda}^{(1)}(t, \theta, \bar{\theta})= & d t \Lambda(t, \theta, \bar{\theta})+d \theta \bar{F}(t, \theta, \bar{\theta}) \\
& +d \bar{\theta} F(t, \theta, \bar{\theta}),
\end{aligned}
$$

in addition to the generalizations in (10). We demand the equality $\tilde{d} \tilde{\lambda}^{(1)}=d \lambda^{(1)}=0$ due to the requirement of the HC. This condition yields the following relationships between the secondary variables and the basic as well as the auxiliary variables of the Lagrangian (1) (see, e.g., [17-19], for details):

$$
\begin{aligned}
R & =\dot{C}, \\
\bar{R} & =\dot{\bar{C}}, \\
S & =\dot{B}, \\
\bar{B}_{2} & =0, \\
B_{1} & =0, \\
S & =0, \\
\bar{B}_{1}+B_{2} & =0, \\
\bar{S} & =0 .
\end{aligned}
$$

If we choose $B_{2}=-\bar{B}_{1}=B$, we obtain the following expansions:

$$
\begin{aligned}
\Lambda^{(h)}(t, \theta, \bar{\theta})= & \lambda(t)+\theta(\dot{\bar{C}})+\bar{\theta}(\dot{C})+\theta \bar{\theta}(i \dot{B}) \\
\equiv & \lambda(t)+\theta\left(s_{a b} \lambda\right)+\bar{\theta}\left(s_{b} \lambda\right) \\
& +\theta \bar{\theta}\left(s_{b} s_{a b} \lambda\right), \\
F^{(h)}(t, \theta, \bar{\theta})= & C(t)+\theta(-i B)+\bar{\theta}(0)+\theta \bar{\theta}(0) \\
\equiv & C(t)+\theta\left(s_{a b} C\right)+\bar{\theta}\left(s_{b} C\right) \\
& +\theta \bar{\theta}\left(s_{b} s_{a b} C\right),
\end{aligned}
$$

$$
\begin{aligned}
\bar{F}^{(h)}(t, \theta, \bar{\theta})= & \bar{C}(t)+\theta(0)+\bar{\theta}(i B)+\theta \bar{\theta}(0) \\
\equiv & \bar{C}(t)+\theta\left(s_{a b} \bar{C}\right)+\bar{\theta}\left(s_{b} \bar{C}\right) \\
& +\theta \bar{\theta}\left(s_{b} s_{a b} \bar{C}\right)
\end{aligned}
$$

where the superscript $(h)$ denotes the expansions of the supervariables after the application of the HC. The above expansions of the supervariables lead to the derivation of proper (i.e., offshell nilpotent and absolutely anticommuting) (anti-)BRST symmetries that have been mentioned in (2). It is to be emphasized that the relationship $\bar{B}_{1}+B_{2}=0$ in (13) is nothing but the CF-condition which turns out to be trivial for our Abelian theory. Furthermore, these expansions imply a relationship between the (anti-)BRST symmetries $s_{(a) b}$ and the translational generators $\left(\partial_{\theta}, \partial_{\bar{\theta}}\right)$ along the Grassmannian directions of the $(1,2)$-dimensional supermanifold. This relationship is as follows: $\lim _{\bar{\theta}=0} \partial_{\theta} \leftrightarrow s_{a b}, \lim _{\theta=0} \partial_{\bar{\theta}} \leftrightarrow s_{b}$. In view of this mapping, it is clear that we have the following equalities; namely,

$$
\begin{gathered}
r(t) \longrightarrow R(t, \theta, \bar{\theta})=r(t), \\
B(t) \longrightarrow \mathscr{B}(t, \theta, \bar{\theta})=B(t), \\
\theta(t) \longrightarrow \Theta(t, \theta, \bar{\theta})=\theta(t), \\
p_{\vartheta}(t) \longrightarrow P_{\vartheta}(t, \theta, \bar{\theta})=p_{\vartheta}(t),
\end{gathered}
$$

because of the fact that we have the (anti-)BRST invariance: $s_{(a) b}\left[r, \vartheta, p_{\vartheta}, B\right]=0$.

We now focus on the GIR that leads to the expansions of $P_{r}(t, \theta, \bar{\theta})$ (in terms of the basic and auxiliary variables) which is the generalizations of $p_{r}(t)$ onto the $(1,2)$-dimensional supermanifold. We observe that $s_{(a) b}\left[\lambda+\dot{p}_{r}\right]=0$. It should be pointed out that all the gauge (i.e., (anti-)BRST) invariant quantities are required to be independent of the Grassmannian variables when they are generalized onto the appropriately chosen supermanifold. This statement implies the following equality under the basic tenets of the augmented version of superfield formalism (see, e.g., [11-14]):

$$
\tilde{\lambda}_{(h)}^{(1)}+\tilde{d} P_{r}(t, \theta, \bar{\theta})=\lambda^{(1)}(t)+d p_{r}(t), \quad d=d t \partial_{t},
$$

where $\tilde{\lambda}_{(h)}^{(1)}(t, \theta, \bar{\theta})=d t \Lambda^{(h)}(t, \theta, \bar{\theta})+d \theta \bar{F}^{(h)}(t, \theta, \bar{\theta})+$ $d \bar{\theta} F^{(h)}(t, \theta, \bar{\theta})$. The super expansions for $\Lambda^{(h)}(t, \theta, \bar{\theta})$, $\bar{F}^{(h)}(t, \theta, \bar{\theta})$, and $F^{(h)}(t, \theta, \bar{\theta})$ are given in (14). Equating the coefficients of $d t, d \theta$, and $d \bar{\theta}$ from the 1.h.s and r.h.s of (16), we obtain the following:

$$
\begin{aligned}
\partial_{\theta} P_{r}(t, \theta, \bar{\theta}) & =-\bar{C}(t)-i \bar{\theta} B(t) \equiv-\bar{F}^{(h)}(t, \theta, \bar{\theta}), \\
\partial_{\bar{\theta}} P_{r}(t, \theta, \bar{\theta}) & =-C(t)+i \theta B(t) \equiv-F^{(h)}(t, \theta, \bar{\theta}), \\
\dot{P}_{r}(t, \theta, \bar{\theta}) & =\dot{p}_{r}(t)+\theta(-\dot{\bar{C}})+\bar{\theta}(-\dot{C})+\theta \bar{\theta}(-i \dot{B}) .
\end{aligned}
$$


It is clear that the solution for the above conditions is the following relationship:

$$
\begin{aligned}
P_{r}(t, \theta, \bar{\theta})= & p_{r}(t)+\theta(-\bar{C})+\bar{\theta}(-C)+\theta \bar{\theta}(-i B) \\
\equiv & p_{r}(t)+\theta\left(s_{a b} p_{r}\right)+\bar{\theta}\left(s_{b} p_{r}\right) \\
& +\theta \bar{\theta}\left(s_{b} s_{a b} p_{r}\right) .
\end{aligned}
$$

A careful observation of (14), (15), and (18) demonstrates that we have already derived the (anti-)BRST symmetry transformations (2) for the $1 \mathrm{D}$ toy model of a rigid rotor. We would like to emphasize that our present method of derivation is totally different from our earlier works [17-19] on the supervariable approach to a $1 \mathrm{D}$ rigid rotor.

We exploit the mathematical power of the $\mathrm{HC}$ in the context of the 2D Abelian 1-form $\left(A^{(1)}=d x^{\mu} A_{\mu}\right)$ gauge theories of the modified versions of the Proca and anomalous gauge theories. In both these theories, we have the basic fields $A_{\mu}(x), C(x)$, and $\bar{C}(x)$ which can be generalized onto an appropriately chosen $(2,2)$-dimensional supermanifold, parametrized by $Z^{M}=\left(x^{\mu}, \theta, \bar{\theta}\right)$. The generalizations of the gauge field $A_{\mu}(x)$ are

$$
\begin{aligned}
A_{\mu}(x) & \longrightarrow \\
B_{\mu}(x, \theta, \bar{\theta}) & =A_{\mu}(x)+\theta \bar{R}_{\mu}(x)+\bar{\theta} R_{\mu}+i \theta \bar{\theta} S_{\mu}
\end{aligned}
$$

and the generalizations of the fields $C(x) \rightarrow F(x, \theta, \bar{\theta}), \bar{C} \rightarrow$ $F(x, \theta, \bar{\theta})$ are given in (10) where we have to replace the parameter $t$ by the $2 \mathrm{D}$ spacetime coordinate $x^{\mu}$ (with $\mu=$ $0,1)$. Furthermore, we have the following generalizations:

$$
\begin{aligned}
d & =d x^{\mu} \partial_{\mu} \longrightarrow \\
\widetilde{d} & =d x^{\mu} \partial_{\mu}+d \theta \partial_{\theta}+d \bar{\theta} \partial_{\bar{\theta}}, \\
A^{(1)} & =d x^{\mu} A_{\mu} \longrightarrow \\
\widetilde{A}^{(1)}(x, \theta, \bar{\theta}) & =d x^{\mu} B_{\mu}(x, \theta, \bar{\theta})+d \theta \bar{F}(x, \theta, \bar{\theta}) \\
& +d \bar{\theta} F(x, \theta, \bar{\theta}),
\end{aligned}
$$

for the exterior derivative to super exterior derivative and the connection 1-form to super connection 1-form on the $(2,2)$ dimensional supermanifold.

The requirement of $\mathrm{HC}$ (i.e., $\tilde{d} \widetilde{A}^{(1)}=d A^{(1)}$ ) yields the following relationship between the secondary fields and basic and auxiliary fields of the Lagrangian densities (3) and (7) for the Abelian 1-form gauge theories under considerations; namely,

$$
\begin{aligned}
R_{\mu} & =\partial_{\mu} C, \\
\bar{R}_{\mu} & =\partial_{\mu} \bar{C}, \\
S_{\mu} & =\partial_{\mu} B, \\
\bar{B}_{1}+B_{2} & =0, \\
\bar{B}_{2} & =B_{1}=0, \\
\bar{B}_{1} & =B=-B_{2} .
\end{aligned}
$$

We observe that $\bar{B}_{1}+B_{2}=0$ is the trivial (anti)-BRST invariant $\mathrm{CF}$-condition which emerges out from our superfield formalism. This is one of the decisive features of our superfield approach. The substitution of these values into the appropriate super expansions yields the following equations (see, e.g., [6-8, 11-14], for details):

$$
\begin{aligned}
B_{\mu}^{(h)}(x, \theta, \bar{\theta})= & A_{\mu}(x)+\theta\left(\partial_{\mu} \bar{C}\right)+\bar{\theta}\left(\partial_{\mu} C\right) \\
& +\theta \bar{\theta}\left(i \partial_{\mu} B\right) \\
\equiv & A_{\mu}+\theta\left(s_{a b} A_{\mu}\right)+\bar{\theta}\left(s_{b} A_{\mu}\right) \\
& +\theta \bar{\theta}\left(s_{b} s_{a b} A_{\mu}\right), \\
F^{(h)}(x, \theta, \bar{\theta})= & C(x)+\theta(-i B)+\bar{\theta}(0)+\theta \bar{\theta}(0) \\
\equiv & C(x)+\theta\left(s_{a b} C\right)+\bar{\theta}\left(s_{b} C\right) \\
& +\theta \bar{\theta}\left(s_{b} s_{a b} C\right), \\
\bar{F}^{(h)}(x, \theta, \bar{\theta})= & \bar{C}(x)+\theta(0)+\bar{\theta}(i B)+\theta \bar{\theta}(0) \\
\equiv & \bar{C}(t)+\theta\left(s_{a b} \bar{C}\right)+\bar{\theta}\left(s_{b} \bar{C}\right) \\
& +\theta \bar{\theta}\left(s_{b} s_{a b} \bar{C}\right),
\end{aligned}
$$

where the superscript $(h)$ denotes the expansions of the superfields after the application of the HC. The expansions (22) are common to all the $2 \mathrm{D}$ theories of our interest. It is clear that we have the mappings: $s_{b} \Leftrightarrow \partial_{\bar{\theta}}, s_{a b} \Leftrightarrow \partial_{\theta}$, as pointed out earlier.

We now focus on the derivation of the (anti-)BRST symmetries for the Stueckelberg field $\phi(x, t)$ of the modified version of the 2D Proca theory. We observe that $s_{(a) b}\left[A_{\mu}-\right.$ $\left.(1 / m) \partial_{\mu} \phi\right]=0$. Thus, we have the following restrictions due to the basic tenets of the augmented version of superfield formalism (see, e.g., [20, 21], for details):

$$
\begin{aligned}
& \widetilde{A}_{(h)}^{(1)}(x, \theta, \bar{\theta})-\frac{1}{m} \widetilde{d} \Phi(x, \theta, \bar{\theta}) \\
& =A^{(1)}(x)-\frac{1}{m} d \phi(x), \quad d=d x^{\mu} \partial_{\mu},
\end{aligned}
$$


where $\widetilde{A}_{(h)}^{(1)}=d x^{\mu} B_{\mu}^{(h)}(x, \theta, \bar{\theta})+d \theta \bar{F}^{(h)}(x, \theta, \bar{\theta})+d \bar{\theta} F^{(h)}(x, \theta, \bar{\theta})$ and $A^{(1)}=d x^{\mu} A_{\mu}(x)$. Exploiting the expansions of (22), we obtain the following conditions:

$$
\begin{aligned}
\partial_{\theta} \Phi(x, \theta, \bar{\theta})= & m \bar{F}^{(h)}(x, \theta, \bar{\theta}) \equiv m \bar{C}(x)+\bar{\theta}(i m B), \\
\partial_{\bar{\theta}} \Phi(x, \theta, \bar{\theta})= & m F^{(h)}(x, \theta, \bar{\theta}) \\
\equiv & m C(x)+\theta(-i m B), \\
\partial_{\mu} \Phi(x, \theta, \bar{\theta})= & \partial_{\mu} \phi(x)+\theta\left(m \partial_{\mu} \bar{C}\right)+\bar{\theta}\left(m \partial_{\mu} C\right) \\
& +\theta \theta\left(i m \partial_{\mu} B\right),
\end{aligned}
$$

from (23). The solution of the above conditions is the following:

$$
\begin{aligned}
\Phi^{(g)}(x, \theta, \bar{\theta})= & \phi(x)+\theta(m \bar{C})+\bar{\theta}(m C)+\theta \bar{\theta}(i m B) \\
\equiv & \phi(x)+\theta\left(s_{a b} \phi\right)+\bar{\theta}\left(s_{b} \phi\right) \\
& +\theta \bar{\theta}\left(s_{b} s_{a b} \phi\right)
\end{aligned}
$$

where the superscript $(g)$ denotes the expansions of the above superfields after the application of the GIR in (23). Thus, it is evident that we have already obtained the (anti-)BRST symmetry transformations for the field $\phi(x)$. The (anti-)BRST invariance $s_{(a) b} B=0$ of the Nakanishi-Lautrup type field $B(x)$ implies that we have $B(x) \rightarrow \mathscr{B}(x, \theta, \bar{\theta})=B(x)$.

A close look at the (anti-)BRST symmetry transformations (6) shows that $s_{(a) b}[\lambda+\dot{\phi}]=0$ and $s_{(a) b}[\lambda+\dot{v}]=0$ in the case of the BRST description of the 2D self-dual bosonic theory. As a consequence, we have the following equality due to the basic tenets of the augmented version of superfield formalism (see, e.g., [22, 23], for details):

$$
\begin{aligned}
& \tilde{\lambda}_{(h)}^{(1)}(x, \theta, \bar{\theta})+\tilde{d} \Phi(x, \theta, \bar{\theta})=\lambda^{(1)}(x)+d \phi(x), \\
& \tilde{\lambda}_{(h)}^{(1)}(x, \theta, \bar{\theta})+\tilde{d} V(x, \theta, \bar{\theta})=\lambda^{(1)}(x)+d v(x),
\end{aligned}
$$

where $d=d t \partial_{t}$ and $\widetilde{d}=d t \partial_{t}+d \theta \partial_{\theta}+d \bar{\theta} \partial_{\bar{\theta}}$. It will be noted that even though our theory is a two $(1+1)$-dimensional theory, it is the coordinate $t$ that plays the role of the evolution parameter [23]. We emphasize that $\tilde{\lambda}_{(h)}^{(1)}=d t \lambda^{(h)}(x, \theta, \bar{\theta})+$ $d \theta \bar{F}^{(h)}(x, \theta, \bar{\theta})+d \bar{\theta} F^{(h)}(x, \theta, \bar{\theta})$ is the same as that in the case of a rigid rotor with the replacement $t \rightarrow x^{\mu}$. It is worthwhile to point out that the $2 \mathrm{D}$ self-dual chiral bosonic theory is different from the $1 \mathrm{D}$ toy model of a rigid rotor because here the basic fields $\lambda, C, \bar{C}$ are functions of the $2 \mathrm{D}$ spacetime coordinates $\left(x^{\mu}\right)$. The equality (26) leads to the following relationships:

$$
\begin{aligned}
\partial_{\theta} V(x, \theta, \bar{\theta}) & \equiv \partial_{\theta} \Phi(x, \theta, \bar{\theta})=-\bar{F}^{(h)}(x, \theta, \bar{\theta}) \\
& =-\bar{C}(x)-i \bar{\theta} B(x), \\
\partial_{\bar{\theta}} V(x, \theta, \bar{\theta}) & \equiv \partial_{\bar{\theta}} \Phi(x, \theta, \bar{\theta})=-F^{(h)}(x, \theta, \bar{\theta}) \\
& =-C(x)+i \theta B(x), \\
\dot{\Phi}(x, \theta \bar{\theta}) & =\dot{\phi}(x)+\theta(-\dot{\bar{C}})+\bar{\theta}(-\dot{C})+\theta \bar{\theta}(-i \dot{B}), \\
\dot{V}(x, \theta \bar{\theta}) & =\dot{v}(x)+\theta(-\dot{\bar{C}})+\bar{\theta}(-\dot{C})+\theta \bar{\theta}(-i \dot{B}) .
\end{aligned}
$$

The above relations imply that we have the following super expansions for the superfields $\Phi(x, \theta, \bar{\theta})$ and $V(x, \theta, \bar{\theta})$ after the application of the GIRs (26); namely,

$$
\begin{aligned}
\Phi^{(g)}(x, \theta, \bar{\theta})= & \phi(x)+\theta(-\bar{C})+\bar{\theta}(-C)+\theta \bar{\theta}(-i B) \\
\equiv & \phi(x)+\theta\left(s_{a b} \phi\right)+\bar{\theta}\left(s_{b} \phi\right) \\
& +\theta \bar{\theta}\left(s_{b} s_{a b} \phi\right), \\
V^{(g)}(x, \theta, \bar{\theta})= & v(x)+\theta(-\bar{C})+\bar{\theta}(-C)+\theta \bar{\theta}(-i B) \\
\equiv & v(x)+\theta\left(s_{a b} v\right)+\bar{\theta}\left(s_{b} v\right) \\
& +\theta \bar{\theta}\left(s_{b} s_{a b} v\right) .
\end{aligned}
$$

Thus, we have determined all the (anti-)BRST symmetry transformations for the self-dual $2 \mathrm{D}$ bosonic theory as is evident from (14) and (28).

Finally, we note that the (anti-)BRST invariant combinations $s_{(a) b}\left[A_{\mu}+\partial_{\mu} \phi\right]=0$ and $s_{(a) b}\left[A_{\mu}-\partial_{\mu} \sigma\right]=0$, in some sense, are the physical quantities in the $2 \mathrm{D}$ modified version of anomalous gauge theory. As a consequence, we have the following equalities due to the basic concepts of the augmented version of superfield approach (see, e.g., [11-14], for details):

$$
\begin{aligned}
& \widetilde{A}_{(h)}^{(1)}(x, \theta, \bar{\theta})+\tilde{d} \Phi(x, \theta, \bar{\theta})=A^{(1)}(x)+d \phi(x), \\
& \widetilde{A}_{(h)}^{(1)}(x, \theta, \bar{\theta})-\tilde{d} \Sigma(x, \theta, \bar{\theta})=A^{(1)}(x)-d \sigma(x),
\end{aligned}
$$

where the 2D fields $\phi(x)$ and $\sigma(x)$ have been generalized to the superfields on the $(2,2)$-dimensional supermanifold as follows: $\phi(x) \rightarrow \Phi(x, \theta, \bar{\theta}), \sigma(x) \rightarrow \Sigma(x, \theta, \bar{\theta})$, and 
$\widetilde{A}_{(h)}^{(1)}=d x^{\mu} B_{\mu}^{(h)}(x, \theta, \bar{\theta})+d \theta \bar{F}^{(h)}(x, \theta, \bar{\theta})+d \bar{\theta} F^{(h)}(x, \theta, \bar{\theta})$. The equalities in (29) lead to the following equations:

$$
\begin{aligned}
\partial_{\theta} \Sigma(x, \theta, \bar{\theta}) \equiv & -\partial_{\theta} \Phi(x, \theta, \bar{\theta})=\bar{F}^{(h)}(x, \theta, \bar{\theta}), \\
\partial_{\bar{\theta}} \Sigma(x, \theta, \bar{\theta}) \equiv & -\partial_{\bar{\theta}} \Phi(x, \theta, \bar{\theta})=F^{(h)}(x, \theta, \bar{\theta}), \\
\partial_{\mu} \Sigma(x, \theta, \bar{\theta})= & \partial_{\mu} \sigma+\theta\left(\partial_{\mu} \bar{C}\right)+\bar{\theta}\left(\partial_{\mu} C\right) \\
& +\theta \bar{\theta}\left(i \partial_{\mu} B\right), \\
\partial_{\mu} \Phi(x, \theta, \bar{\theta})= & \partial_{\mu} \phi+\theta\left(-\partial_{\mu} \bar{C}\right)+\bar{\theta}\left(-\partial_{\mu} C\right) \\
& +\theta \bar{\theta}\left(-i \partial_{\mu} B\right) .
\end{aligned}
$$

The above conditions are satisfied by the following explicit super expansions for the super fields (corresponding to the ordinary fields $\phi(x)$ and $\sigma(x))$; namely,

$$
\begin{aligned}
\Phi^{(g)}(x, \theta, \bar{\theta})= & \phi(x)+\theta(-\bar{C})+\bar{\theta}(-C)+\theta \bar{\theta}(-i B) \\
\equiv & \phi(x)+\theta\left(s_{a b} \phi\right)+\bar{\theta}\left(s_{b} \phi\right) \\
& +\theta \bar{\theta}\left(s_{b} s_{a b} \phi\right), \\
\Sigma^{(g)}(x, \theta, \bar{\theta})= & \sigma(x)+\theta(\bar{C})+\bar{\theta}(C)+\theta \bar{\theta}(i B) \\
\equiv & \sigma(x)+\theta\left(s_{a b} \sigma(x)\right)+\bar{\theta}\left(s_{b} \sigma(x)\right) \\
& +\theta \bar{\theta}\left(s_{b} s_{a b} \sigma(x)\right),
\end{aligned}
$$

where the superscript $(g)$ denotes the super expansions obtained after the application of the GIRs mentioned in (29). Finally, we know that $s_{(a) b} B(x)=0$. As a consequence, it is evident that we have the generalization: $B(x) \rightarrow \mathscr{B}(x, \theta, \bar{\theta})=$ $B(x)$. A close and careful look at (22) and (31) demonstrates that we have obtained all the (anti-)BRST transformations for all the fields of the 2D modified version of the anomalous gauge theory.

\section{SUSP Unitary Operator: Universality Aspects}

We establish, in this section, that the form of the SUSP unitarity operator (and its Hermitian conjugate) is the same for all the Abelian models we have discussed in our present investigation and the $4 \mathrm{D}$ interacting $U(1)$ gauge theory with Dirac and complex scalar fields that have been discussed in our earlier work [15] where we have derived the explicit form of these operators (for the electric charge $e=1$ ) as (see, e.g., [15], for details)

$$
\begin{aligned}
U(x, \theta, \bar{\theta}) & =1+\theta(-i \bar{C})+\bar{\theta}(-i C)+\theta \bar{\theta}(B-C \bar{C}), \\
U^{\dagger}(x, \theta, \bar{\theta}) & =1+\theta(i \bar{C})+\bar{\theta}(i C)+\theta \bar{\theta}(-B+\bar{C} C) .
\end{aligned}
$$

The above unitarity operators can be exponentiated as follows [15]:

$$
\begin{aligned}
U(x, \theta, \bar{\theta}) & =\exp [\theta(-i \bar{C})+\bar{\theta}(-i C)+\theta \bar{\theta} B], \\
U^{\dagger}(x, \theta, \bar{\theta}) & =\exp [\theta(i \bar{C})+\bar{\theta}(i C)-\theta \bar{\theta} B] .
\end{aligned}
$$

These forms of the unitary operators explicitly show the Abelian $U(1)$ group structure in the transformation superspace of our theory and it is elementary to check that $U U^{\dagger}=$ $U^{\dagger} U=1$. The latter can be also checked from the explicit expansions of $U(x, \theta, \bar{\theta})$ and $U^{\dagger}(x, \theta, \bar{\theta})$ which are quoted in (32). In fact, the group structure becomes clear due to (33).

In the case of $1 \mathrm{D}$ toy model of a rigid rotor, it is very interesting to note that the $\mathrm{HC}$ can be expressed as follows:

$$
\begin{aligned}
\tilde{\lambda}_{(h)}^{(1)}= & U(t, \theta, \bar{\theta}) \lambda^{(1)}(t) U^{\dagger}(t, \theta, \bar{\theta}) \\
& +i \tilde{d} U(t, \theta, \bar{\theta}) U^{\dagger}(t, \theta, \bar{\theta})
\end{aligned}
$$

where the expression for $U(t, \theta, \bar{\theta})$ is the same as that given in (32) and (33) with the replacement: $\left.U(x, \theta, \bar{\theta})\right|_{x=t} \equiv U(t, \theta, \bar{\theta})$, and the other symbols in (34) have already been expalined earlier. It is worthwhile to mention that relationship (34) is just the $1 \mathrm{D}$ version of exactly the same kind of relationship (cf. (40) below) obtained in our earlier work on the interacting 4D Abelian $U(1)$ gauge theory with Dirac and complex scalar fields [15]. We can readily check that (34) provides an alternative to the $\mathrm{HC}\left(\tilde{d} \tilde{\lambda}_{(h)}^{(1)}=d \lambda^{(1)}\right)$ because we get the exact form of the super expansions (14) which have already been obtained earlier in Section 3. Furthermore, our equation (34) also provides the reason behind the application of the $\mathrm{HC}$ because it can be explicitly checked that

$$
\tilde{d} \tilde{\lambda}_{(h)}^{(1)}=\tilde{d} \lambda^{(1)}(t)-i \tilde{d} U(t, \theta, \bar{\theta}) \wedge \tilde{d} U^{\dagger}(t, \theta, \bar{\theta}),
$$

where we have applied the operator $\widetilde{d}=d t \partial_{t}+d \theta \partial_{\theta}+$ $d \bar{\theta} \partial_{\bar{\theta}}$ from the left on (34) and used the basic property of the differential geometry ((i.e., $\left.\left.(\tilde{d})^{2}=0\right)\right)$. We note that $\tilde{d} \tilde{\lambda}^{(1)}(t)=\tilde{d} \lambda^{(1)}(t)$ (as $\partial_{\theta} \lambda^{(1)}(t)=\partial_{\bar{\theta}} \lambda^{(1)}(t)=0$ ) and it is the forms of $U$ and $U^{\dagger}$ in (33) that show that $\left(\tilde{d} U \wedge \tilde{d} U^{\dagger}=0\right)$. This statement can be also verified by the explicit expansions as listed in the following:

$$
\begin{aligned}
\tilde{d} U= & d t[\theta(-i \dot{\bar{C}})+\bar{\theta}(-i \dot{C})+\theta \bar{\theta}(\dot{B}-\dot{\bar{C}} \bar{C}-C \dot{\bar{C}})] \\
& +d \theta[-i \bar{C}+\bar{\theta}(B-C \bar{C})] \\
& +d \bar{\theta}[-i C-\theta(B-C \bar{C})], \\
\widetilde{d} U^{\dagger}= & d t[\theta(i \dot{\bar{C}})+\bar{\theta}(i \dot{C})+\theta \bar{\theta}(-\dot{B}+\dot{\bar{C}} C+\bar{C} \dot{C})] \\
& +d \theta[i \bar{C}+\bar{\theta}(-B+\bar{C} C)] \\
& +d \bar{\theta}[i C-\theta(-B+\bar{C} C)] .
\end{aligned}
$$


We can collect the coefficients of $(d t \wedge d \theta),(d \theta \wedge d \bar{\theta}),(d \theta \wedge d \theta)$, $(d \theta \wedge d \bar{\theta})$, and $(d \bar{\theta} \wedge d \bar{\theta})$ in $\left(\widetilde{d} U \wedge \tilde{d} U^{\dagger}\right)$. Interestingly, these coefficients turn out to be exactly zero. This observation, once again, proves that $\widetilde{d} U \wedge \widetilde{d} U^{\dagger}=0$, where, of course, we have used the input $d t \wedge d t=0$.

The GIRs (i.e., (anti-)BRST) restrictions $s_{(a) b}\left[\lambda+\dot{p}_{r}\right]=0$ have been exploited to derive the expansion for $P_{r}^{(g)}(t, \theta, \bar{\theta})$ in (18). This relationship can be also expressed in terms of the unitary operators (with $p_{r}(t) \rightarrow P_{r}(t, \theta, \bar{\theta})$ ) because the equality

$$
\widetilde{d} P_{r}(t, \theta, \bar{\theta})+\tilde{\lambda}_{(h)}^{(1)}(t, \theta, \bar{\theta})=d p_{r}(t)+\lambda^{(1)}(t)
$$

leads to the following relationships when we use (34) in it; namely,

$$
\begin{aligned}
& \partial_{\theta} P_{r}(t, \theta, \bar{\theta})=-i\left(\partial_{\theta} U\right) U^{\dagger} \equiv-\bar{F}^{(h)}(t, \theta, \bar{\theta}), \\
& \partial_{\bar{\theta}} P_{r}(t, \theta, \bar{\theta})=-i\left(\partial_{\bar{\theta}} U\right) U^{\dagger} \equiv-F^{(h)}(t, \theta, \bar{\theta}), \\
& \partial_{t} P_{r}(t, \theta, \bar{\theta})=\partial_{t} p_{r}-i\left(\partial_{t} U\right) U^{\dagger} .
\end{aligned}
$$

The last relationship yields the following explicit equation:

$$
\dot{P}_{r}(t, \theta, \bar{\theta})=\dot{p}_{r}(t)+\theta(-\dot{\bar{C}})+\bar{\theta}(-\dot{C})+\theta \bar{\theta}(-i \dot{B}) .
$$

The solution of (38) and (39) is nothing but the expansion given in (18) which leads to the derivation of the (anti-)BRST symmetry transformations for $p_{r}(t)$.

For the modified versions of the 2D Proca and anomalous Abelian 1-form gauge theories, we have the following form of the HC in terms of the SUSP unitary operators; namely,

$$
\begin{aligned}
\widetilde{A}_{(h)}^{(1)}(x, \theta, \bar{\theta})= & U(x, \theta, \bar{\theta}) A^{(1)}(x) U^{\dagger}(x, \theta, \bar{\theta}) \\
& +i(\tilde{d} U(x, \theta, \bar{\theta})) U^{\dagger}(x, \theta, \bar{\theta}),
\end{aligned}
$$

where the forms of $U(x, \theta, \bar{\theta})$ and $U^{\dagger}(x, \theta, \bar{\theta})$ are quoted in (32) and (33). We wish to lay emphasis on the fact that the above relationship has been obtained in the $4 \mathrm{D}$ interacting Abelian 1-form U(1) gauge theory with Dirac and complex scalar fields where the concept of the covariant derivatives plays an important role. The point to be noted (and emphasized) is the observation that relationship (40) is valid for all kinds of Abelian 1-form gauge theory where even the covariant derivatives are not explicitly defined. The substitution of the explicit forms of SUSP unitary operators from (32) and (33) into (40) produces the expansions that are given in (22) where one has to take into account the definition of the super 1-form: $\widetilde{A}_{(h)}^{(1)}=d x^{\mu} B_{\mu}^{(h)}(x, \theta, \bar{\theta})+d \theta \bar{F}^{(h)}(x, \theta, \bar{\theta})+$ $d \bar{\theta} F^{(h)}(x, \theta, \bar{\theta})$ on the l.h.s.

The existence of SUSP unitary operators and its Hermitian conjugate also provides the logical reason behind the imposition of the HC (cf. Section 3) as it can be shown that

$$
\tilde{d} \widetilde{A}_{(h)}^{(1)}=\tilde{d} A^{(1)}(x)-i(\tilde{d} U) \wedge\left(\tilde{d} U^{\dagger}\right) .
$$

The above equation emerges out from (40) when we apply a super exterior derivative $\widetilde{d}$ from the left on it. It is evident that $\widetilde{d} A^{(1)}(x)=d A^{(1)}(x)$ and $(\widetilde{d} U) \wedge\left(\widetilde{d} U^{\dagger}\right)=0$ due to the form of $U(x, \theta, \bar{\theta})$ and $U^{\dagger}(x, \theta, \bar{\theta})$ in (33). One can explicitly compute the expression from $(\widetilde{d} U) \wedge\left(\widetilde{d} U^{\dagger}\right)$ and collect the coefficients of $\left(d x^{\mu} \wedge d x^{\nu}\right),\left(d x^{\mu} \wedge d \theta\right),\left(d x^{\mu} \wedge d \bar{\theta}\right),(d \theta \wedge d \theta),(d \bar{\theta} \wedge d \bar{\theta})$, and $(d \theta \wedge d \bar{\theta})$ to prove that all these coeficients are zero. Thus, we finally obtain the HC as follows: $\widetilde{d} \widetilde{A}_{(h)}^{(1)}=d A^{(1)}(x)$ which primarily emerges out from (40) that is expressed in terms of the SUSP unitary operators $U(x, \theta, \bar{\theta})$ and $U^{\dagger}(x, \theta, \bar{\theta})$. The key equations (40), (41) and the HC $\left(\tilde{d} \widetilde{A}^{(1)}=d A^{(1)}\right)$ are common features to the BRST description of the 2D modified versions of Proca and anomalous gauge theories. On the other hand, the $1 \mathrm{D}$ rigid rotor and 2D self-dual field theory are described by relationships (34) and (35) (with $\tilde{d} \tilde{\lambda}_{(h)}^{(1)}=d \lambda^{(1)}$ ). However, in the case of the 2D self-dual theory, one has to replace $t$ by $x^{\mu}(\mu=0,1)$ in the expression for the gauge variable $\lambda(t)$ (e.g., $\lambda(t) \rightarrow \lambda(x, t))$.

We are now in the position to express the GIR in the language of SUSP unitary operator and its Hermitian conjugate. First of all, we focus on the modified version of 2D Proca theory and exploit the relationship given in (23). Using the relationship of (40), we obtain the following:

$$
\begin{aligned}
& \partial_{\theta} \Phi(x, \theta, \bar{\theta})=i m\left(\partial_{\theta} U\right) U^{\dagger}, \\
& \partial_{\bar{\theta}} \Phi(x, \theta, \bar{\theta})=i m\left(\partial_{\bar{\theta}} U\right) U^{\dagger}, \\
& \partial_{\mu} \Phi(x, \theta, \bar{\theta})=\partial_{\mu} \phi(x)+i m\left(\partial_{\mu} U\right) U^{\dagger},
\end{aligned}
$$

in the language of the SUSP unitary operator and its Hermitian conjugate. The substitution of the explicit form of $U(x, \theta, \bar{\theta})$ and $U^{\dagger}(x, \theta, \bar{\theta})$ from (32) leads to the solution of (42) as given in (25) (see Section 3). As far as the GIRs (26) for the $2 \mathrm{D}$ self-dual theory are concerned, we can express these in terms of the SUSP unitary operators as follows:

$$
\begin{aligned}
& \partial_{\theta} V(x, \theta, \bar{\theta}) \equiv \partial_{\theta} \Phi(x, \theta, \bar{\theta})=-i\left(\partial_{\theta} U\right) U^{\dagger} \\
& \partial_{\bar{\theta}} V(x, \theta, \bar{\theta}) \equiv \partial_{\bar{\theta}} \Phi(x, \theta, \bar{\theta})=-i\left(\partial_{\bar{\theta}} U\right) U^{\dagger} \\
& \partial_{t} V(x, \theta, \bar{\theta})=\partial_{t} v(x)-i\left(\partial_{t} U\right) U^{\dagger} \\
& \partial_{t} \Phi(x, \theta, \bar{\theta})=\partial_{t} \phi(x)-i\left(\partial_{t} U\right) U^{\dagger}
\end{aligned}
$$

where we have used the definition $\widetilde{d}=d t \partial_{t}+d \theta \partial_{\theta}+d \bar{\theta} \partial_{\bar{\theta}}$ in (26) because the evolution parameter of this $2 \mathrm{D}$ theory is $t$ only (see, e.g., [23], for details). The explicit substitution of $U(x, \theta, \bar{\theta})$ and $U^{\dagger}(x, \theta, \bar{\theta})$ into (43) yields relation (27) whose solution is (28) in terms of the (anti-)BRST symmetry transformations. 
Finally, we concentrate on the GIRs listed in (29) for the $2 \mathrm{D}$ modified version of the anomalous gauge theory. Using (40) in (29), we obtain the following:

$$
\begin{aligned}
& \partial_{\theta} \Sigma(x, \theta, \bar{\theta}) \equiv-\partial_{\theta} \Phi(x, \theta, \bar{\theta})=i\left(\partial_{\theta} U\right) U^{\dagger}, \\
& \partial_{\bar{\theta}} \Sigma(x, \theta, \bar{\theta}) \equiv-\partial_{\bar{\theta}} \Phi(x, \theta, \bar{\theta})=i\left(\partial_{\bar{\theta}} U\right) U^{\dagger}, \\
& \partial_{\mu} \Sigma(x, \theta, \bar{\theta})=\partial_{\mu} \sigma(x)+i\left(\partial_{\mu} U\right) U^{\dagger}, \\
& \partial_{\mu} \Phi(x, \theta, \bar{\theta})=\partial_{\mu} \phi(x)-i\left(\partial_{\mu} U\right) U^{\dagger} .
\end{aligned}
$$

It is straightforward to check that the r.h.s. of (44) yields the r.h.s. of (30) whose solution is (31). Thus, we conclude that the GIRs, used in the cases of the 1D and 2D models under consideration, can all be expressed in terms of the SUSP unitary operator and its Hermitian conjugate. As a consequence, these operators do provide the alternatives to the HC and the GIRs that are exploited in the derivation of the full set of (anti-)BRST symmetries for the models under consideration.

\section{Conclusions}

We have established, in our present investigation, that the SUSP unitary operator and its Hermitian conjugate have a universal mathematical expression which is true for different kinds of $U(1)$ Abelian 1-form gauge theories. In the case of $4 \mathrm{D}$ interacting Abelian $U(1)$ gauge theory with the Dirac and complex scalar fields (where the covariant derivatives are explicitly defined), the SUSP unitary operator and its Hermitian conjugate have been derived explicitly within the framework of augmented version of superfield formalism [15]. It turns out that this mathematical form of the above operators remains relevant and correct even in the context of the Abelian 1-form gauge theories in one $(0+1)$ dimension and two $(1+1)$ dimensions of spacetime, too, where the covariant derivatives are not defined in an explicit manner. The observation of the universality of these operators, we reemphasize, is one of the highlights of our present investigation.

In our present endeavor, we have applied the augmented version of superfield formalism [15] to derive the proper (i.e., off-shell nilpotent and absolutely anticommuting) (anti-)BRST symmetry transformations for a new system of the $2 \mathrm{D}$ modified version of anomalous gauge theory (see Section 3). Thus, it is a novel result in our present investigation. We have established that our superfield approach could be applied to the new systems of field theories and/or the toy models of gauge theories and we can be sure of obtaining the proper (anti-)BRST symmetry transformations. The key ingredients that play a decisive role in these derivations are the celebrated HC and GIRs. One of the key features of our superfield approach is the emergence of the CF-condition (cf. (13), (21)), which turns out to be trivial in the case of Abelian 1-form gauge theories of different varieties (cf. (13), (21)).

The toy model and the field theoretical examples, considered in our present investigation, are interesting and instructive because their internal symmetries provide the physical realizations of the de Rham cohomological operators of differential geometry within the framework of the BRST formalism. For these models of gauge theories [17-23], there exist six continuous symmetries and a couple of discrete symmetries which provide the analogues of the cohomological operators, the Hodge duality operation, the properties of the differential operators, and so forth, in a physical manner (see, e.g., [17-23], for details). Thus, these models have a whole lot of richness (as far as the mathematical and physical aspects of a well-defined physical theory in any arbitrary dimension of spacetime are concerned).

It would be a very nice future endeavor to establish the universality of the SUSP operators in the context of some models of the non-Abelian nature. The models that we have considered in our present investigation are examples of Hodge theory where the proper (anti-)co-BRST symmetries also exist. Thus, it would be a challenging problem for us to derive the SUSP unitary operator and its Hermitian conjugate corresponding to these proper continuous symmetry transformations. We hope that the discrete symmetry transformations in our models for the Hodge theory (corresponding to the Hodge duality $(*)$ operation) would be able to lead us to derive these SUSP unitary operators in a consistent and cogent manner. It would be also interesting to obtain such operators in the context of diffeomorphism invariant theories. We are currently busy with such kind of issues which we hope to resolve in our future publications.

\section{Conflict of Interests}

The authors declare that there is no conflict of interests regarding the publication of this paper.

\section{Acknowledgments}

One of the authors (T. Bhanja) would like to gratefully acknowledge the financial support from CSIR, Government of India, New Delhi, under its SRF-scheme. Another author (N. Srinivas) is thankful to the BHU-fellowship for financial support. The present investigation has been carried out under the above financial supports.

\section{References}

[1] J. Thierry-Mieg, "Geometrical reinterpretation of FaddeevPopov ghost particles and BRS transformations," Journal of Mathematical Physics, vol. 21, article 2834, 1980.

[2] M. Quirós, F. J. De Urries, J. Hoyos, M. L. Mazón, and E. Rodriguez, "Geometrical structure of Faddeev-Popov fields and invariance properties of gauge theories," Journal of Mathematical Physics, vol. 22, no. 8, pp. 1767-1774, 1981.

[3] R. Delbourgo and P. D. Jarvis, "Extended BRS invariance and OSp (4/2) supersymmetry supersymmetry," Journal of Physics $A$, vol. 15 , no. 2 , p. $611,1982$.

[4] L. Bonora and M. Tonin, "Superfield formulation of extended BRS symmetry," Physics Letters B, vol. 98, no. 1-2, pp. 48-50, 1981. 
[5] L. Bonora, P. Pasti, and M. Tonin, "Extended BRS symmetry in non-Abelian gauge theories," Il Nuovo Cimento A, vol. 64, no. 3, pp. 307-331, 1981.

[6] L. Bonora, P. Pasti, and M. Tonin, "Superspace approach to quantum gauge theories," Annals of Physics, vol. 144, no. 1, pp. 15-33, 1982.

[7] L. Baulieu and J. Thierry-Mieg, "The principle of BRS symmetry: an alternative approach to Yang-Mills theories," Nuclear Physics B, vol. 197, no. 3, pp. 477-508, 1982.

[8] L. Alvarez-Gaumé and L. Baulieu, "The two quantum symmetries associated with a classical symmetry," Nuclear Physics B, vol. 212, no. 2, pp. 255-267, 1983.

[9] R. P. Malik, "One-form Abelian gauge theory as the Hodge theory," International Journal of Modern Physics A, vol. 22, no. 21, pp. 3521-3562, 2007.

[10] R. Kumar, S. Krishna, A. Shukla, and R. P. Malik, "Abelian pform $(p=1,2,3)$ gauge theories as the field theoretic models for the Hodge theory," International Journal of Modern Physics A, vol. 29, no. 24, Article ID 1450135, 2014.

[11] R. P. Malik, "Abelian 2-form gauge theory: superfield formalism," The European Physical Journal C, vol. 60, no. 3, pp. 457470, 2009.

[12] R. P. Malik, "Superfield approach to nilpotent symmetries for QED from a single restriction: an alternative to the horizontality condition," Journal of Physics A: Mathematical and General, vol. 39, no. 33, p. 10575, 2006.

[13] R. P. Malik, "Unique nilpotent symmetry transformations for matter fields in QED: augmented superfield formalism," The European Physical Journal C, vol. 47, no. 1, pp. 227-234, 2006.

[14] R. P. Malik, "Nilpotent symmetries for a spinning relativistic particlein augmented superfield formalism," The European Physical Journal C, vol. 45, no. 2, pp. 513-524, 2006.

[15] D. Shukla, T. Bhanja, and R. P. Malik, "Superspace unitary operator in QED with Dirac and complex scalar fields: superfield approach," Europhysics Letters, vol. 112, no. 1, Article ID 11001, 2015.

[16] T. Bhanja, D. Shukla, and R. P. Malik, "Superfield approach to non-Abelian gauge theory with dirac fields: superspace unitaryoperator," http://arxiv.org/abs/1509.07319.

[17] S. Gupta and R. P. Malik, "Rigid rotor as a toy model for Hodge theory," The European Physical Journal C, vol. 68, no. 1, pp. 325335,2010

[18] D. Shukla, T. Bhanja, and R. P. Malik, "Canonical brackets of a toy model for the Hodge theory without its canonical conjugate momenta," International Journal of Modern Physics A, vol. 30, Article ID 1550115, 2015.

[19] D. Shukla, T. Bhanja, and R. P. Malik, "Nilpotent symmetries of a rigid rotor: supervariableapproach," http://arxiv.org/abs/ 1407.6574

[20] T. Bhanja, D. Shukla, and R. P. Malik, "Novel symmetries in the modified version of two dimensional Proca theory," European Physical Journal C, vol. 73, article 2535, 2013.

[21] A. Shukla, S. Krishna, and R. P. Malik, "Augmented superfield approach to nilpotent symmetries of the modified version of $2 \mathrm{D}$ Proca theory," Advances in High Energy Physics, vol. 2015, Article ID 258536, 21 pages, 2015.

[22] D. Shukla, T. Bhanja, and R. P. Malik, "Self-dual chiral boson: augmented superfield approach," The European Physical Journal C, vol. 74, article 3025, 2014.

[23] T. Bhanja, N. Srinivas, and R. P. Malik, "(Anti-)BRST and (Anti-)co-BRST symmetries of a system ofrigid rotor: augmented supervariable approach," http://arxiv.org/abs/1504.04237.
[24] D. Nemeschansky, C. Preitschopf, and M. Weinstein, "A BRST primer," Annals of Physics, vol. 183, no. 2, pp. 226-268, 1988.

[25] S. Gupta, R. Kumar, and R. P. Malik, "On free 4D Abelian 2form and anomalous 2D Abelian 1-form gauge theories," The European Physical Journal C, vol. 65, no. 1-2, pp. 311-329, 2010. 

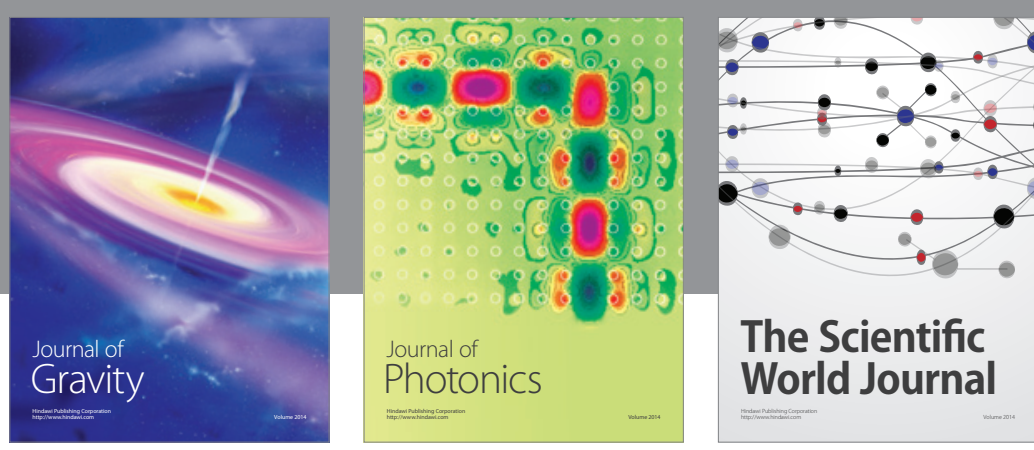

The Scientific World Journal
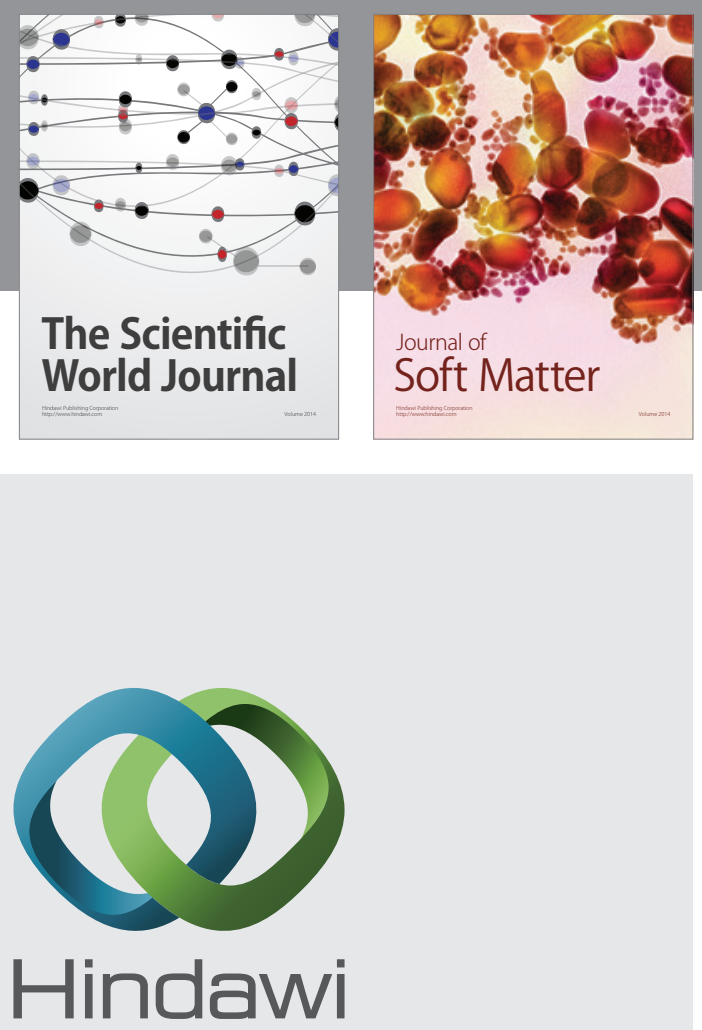

Submit your manuscripts at

http://www.hindawi.com

nternational Journal of

Statistical Mechanics
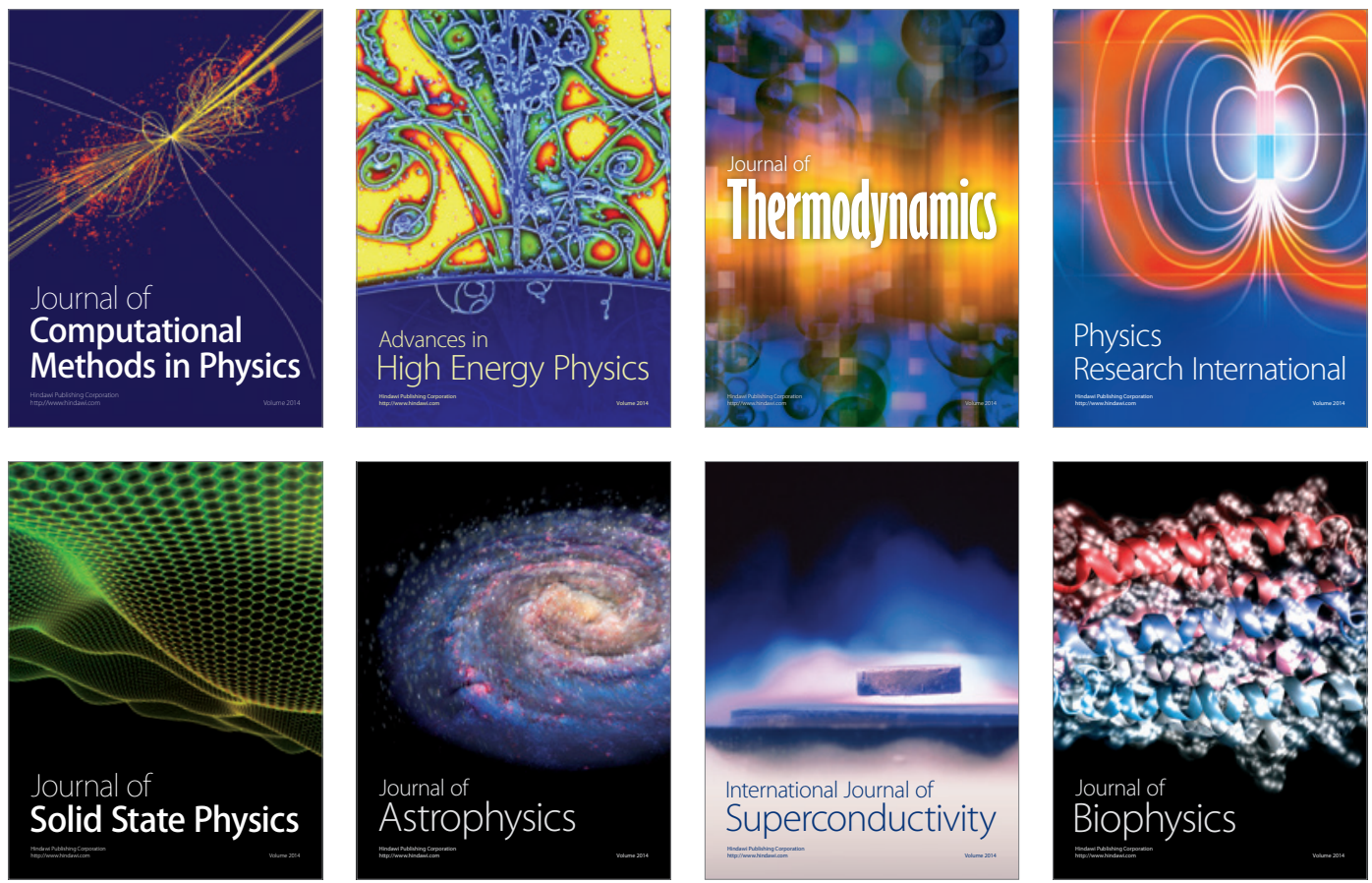
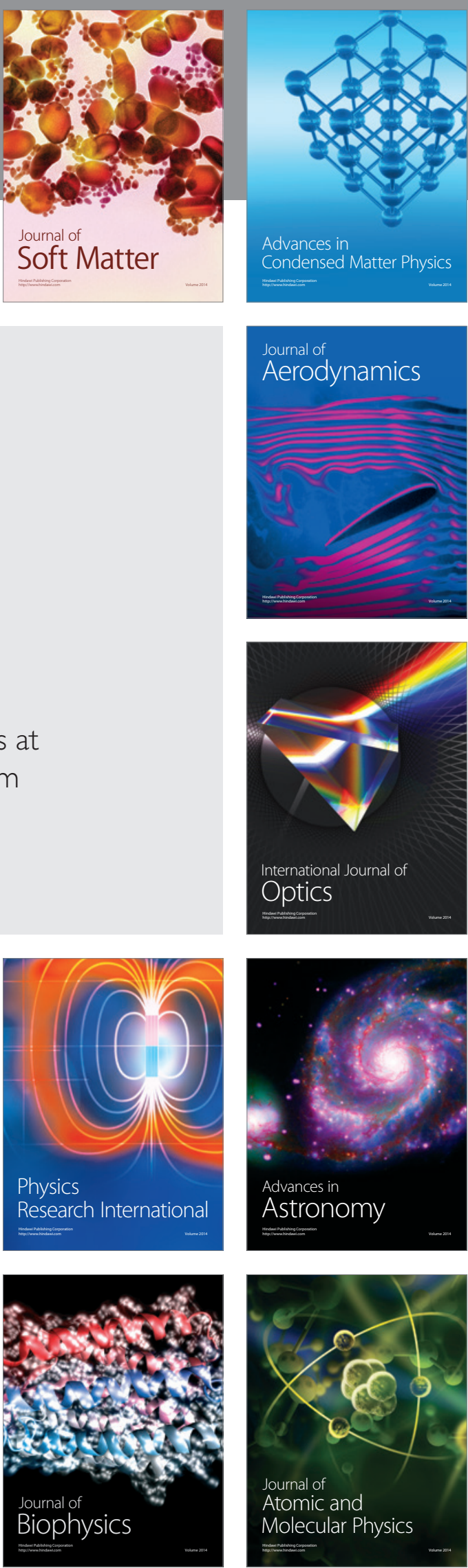